\title{
QUALIDADE DE VIDA E FATORES ASSOCIADOS EM APOSENTADOS POR INVALIDEZ DE UMA UNIVERSIDADE PÚBLICA BRASILEIRA ${ }^{1}$
}

\author{
QUALITY OF LIFE AND ASSOCIATED FACTORS IN RETIREES DUE TO \\ DISABILITY IN A BRAZILIAN PUBLIC UNIVERSITY
}

\section{CALIDAD DE VIDA Y FACTORES ASOCIADOS EN JUBILADOS POR INVALIDEZ DE UNA UNIVERSIDAD PÚBLICA BRASILEÑA}

\author{
Aline Aparecida Oliveira Moreira* \\ Júlia Trevisan Martins ${ }^{* *}$ \\ Maria Lúcia do Carmo Cruz Robazzi ${ }^{* * *}$ \\ Renata Perfeito Ribeiro ${ }^{* * * *}$ \\ Marcela Maria Birolim ${ }^{* * * * *}$ \\ Maria José Quina GaLdino ${ }^{* * * * *}$
}

RESUMO

Objetivo: Avaliar a Qualidade de Vida e os fatores associados em servidores aposentados por invalidez de uma universidade pública. Material e método: Estudo analítico realizado com 40 servidores aposentados por invalidez entre 2000 e 2014 de uma universidade pública brasileira. Foi utilizado um questionário para obtenção dos dados de caracterização dos participantes e o Short Form Health Survey (SF36v2) para avaliar a qualidade de vida. Para análise estatística utilizou-se regressão linear múltipla pela técnica de Bootstrap. Resultados: Os aposentados apresentaram escores médios de qualidade de vida de 42,3 (DP 12,1) no componente físico e 41,1 (DP $16,4)$ no mental. As menores percepções nos domínios de qualidade de vida estiveram associadas sobretudo às doenças crônicas, enquanto que as melhores percepções de alguns domínios foram associadas ao lazer e possuir relacionamento conjugal. Conclusão: Os participantes apresentaram uma qualidade de vida insatisfatória. Faz-se necessário o controle das doenças crônicas, estímulo aos hábitos de vida saudáveis e promoção de saúde, a fim de melhorar a qualidade de vida destas pessoas.

Palavras chave: Aposentadoria, qualidade de vida, seguro por invalidez, universidade.

\footnotetext{
${ }^{1}$ Moreira AAO. Qualidade de vida entre servidores aposentados por invalidez de uma universidade pública [dissertação]. Mestrado em Enfermagem. Universidade Estadual de Londrina; 2016.

*Enfermeira. Mestre em Enfermagem. Hospital Zona Sul de Londrina. Londrina, Paraná, Brasil. Email: alineaoliveira@ yahoo.com.br.

${ }^{* *}$ Enfermeira. Doutora em Enfermagem. Professor Associado da Universidade Estadual de Londrina. Londrina, Paraná, Brasil.Email: jtmartins@uel.br

${ }^{* * *}$ Enfermeira. Doutora em Enfermagem. Professor Titular da Escola de Enfermagem de Ribeirão Preto da Universidade de São Paulo. Email: avrmlccr@eerp.usp.br

${ }^{* * * *}$ Enfermeira. Doutora em Enfermagem. Professor Adjunto da Universidade Estadual de Londrina. Londrina, Paraná, Brasil. E-mail: perfeitorenata@gmail.com

${ }^{* * * * *}$ Enfermeira. Pós-Doutora em Enfermagem. Universidade Estadual de Londrina. Londrina, Paraná, Brasil. Email: marcelabirolim@hotmail.com

${ }_{* * * * * *}$ Enfermeira. Doutoranda no Programa de Pós-Graduação em Enfermagem da Universidade Estadual de Maringá. Maringá, Paraná, Brasil. Professor Assistente da Universidade Estadual do Norte do Paraná. Bandeirantes, Paraná, Brasil. Email: mariagaldino@uenp.edu.br. Autor correspondente.
} 


\begin{abstract}
Objective: To evaluate the Quality of Life and the associated factors in civil servants retired due to disability. Method: Analytical study with 40 retired public employees due to disability from a Brazilian public university between the years 2000-2014. A questionnaire was used to obtain the data characterizing the participants, and the Short Form Health Survey (SF36v2) was used to evaluate their quality of life. For statistical analysis, multiple linear regression and bootstrapping were used. Results: Disabled retirees presented mean scores of 42.3 (DS 12.1) in the physical component and 41.1 (DS 16.4) in the mental component. The lower perceptions in the domains of quality of life were mainly associated with chronic disease, while the highest perceptions of some domains were associated with leisure and being in a marital relationship. Conclusion: The participants presented an unsatisfactory quality of life. It is necessary to control chronic diseases, stimulate healthy living habits and promote health in order to improve the quality of life of these people.
\end{abstract}

Key words: Retirement, quality of life, insurance, disability, universities.

\title{
RESUMEN
}

Objetivo: Evaluar la Calidad de Vida y los factores asociados en los empleados jubilados por incapacidad de una universidad pública. Material y método: Estudio analítico con 40 empleados jubilados por discapacidad entre los años 2000-2014 de una universidad pública brasileña. Se utilizó un cuestionario para la obtención de los datos de caracterización de los participantes y Short Form Health Survey (SF36v2) para evaluar la calidad de vida. El análisis estadístico se realizó mediante regresión lineal múltiple por Bootstrap. Resultados: Los jubilados por discapacidad tuvieron puntuaciones medias en calidad de vida de 42,3 (DT 12,1) en el componente físico y 41,1 (DT 16,4) en el mental. Percepciones menores sobre la calidad de vida en los dominios estaban principalmente relacionados con enfermedades crónicas, mientras que las mejores percepciones de algunas áreas fueron asociadas con el ocio y tener relación conyugal. Conclusión: Los participantes tuvieron una insatisfactoria calidad de vida. Es necesario el control de enfermedades crónicas, estimulación de hábitos de vida saludables y promoción de salud para mejorar la calidad de vida de estas personas.

Palabras clave: Jubilación, calidad de vida, seguro por discapacidad, universidades.

Fecha recepción: 13/02/17 Fecha aceptación: 25/04/18

\section{INTRODUÇÃO}

Envelhecer é um processo natural, no qual as capacidades de adaptação das pessoas vão diminuindo diante das alterações biológicas, emocionais e sociais vivenciadas (1). A senescência está associada à aposentadoria e também representa um evento na vida do trabalhador e traz mudanças importantes de natureza biopsicossocial e financeira, assim é necessário um planejamento prévio. Desta forma, a soma das mudanças vivenciadas no envelhecimento e decorrentes da transição para a aposentadoria pode interferir na qualidade de vida (QV) do indivíduo (2).

A QV é um construto humano, que reflete o momento histórico, social e cultural ao qual o homem pertence. Quando incluído à saúde, une-se ao conceito a construção social coletiva dos padrões que as diversas sociedades estabelecem para si (3). Muitos são os conceitos de QV, porém uma definição amplamente difundida é a da Organização Mundial da Saúde, elaborada a partir de um grupo de especialistas de diferentes países, em que a QV foi definida como "a percepção que a pessoa tem sobre si mesma, conside- 
rando a cultura e valores nos quais está inserida, elaborando seus objetivos, com suas expectativas, padrões e preocupações" (4).

Derivada da QV encontra-se a qualidade de vida no trabalho (QVT) que permeia não apenas os indivíduos como também as organizações onde estão inseridos, já que o trabalho e seu ambiente interferem na QV em decorrência dos riscos laborais. A gestão das instituições pode promover a QVT por meio da organização do trabalho, integração social, motivação, adoção de medidas de saúde e segurança. A adoção de práticas voltadas para QVT contribui para diminuição do absenteísmo, inclusive as decorrentes do adoecimento (5).

A QV tem sido objeto de muitos estudos e, por sua vez, tem influenciado as políticas públicas, visto que, é um indicador importante da eficácia e do impacto de vários tratamentos, da comparação de procedimentos para o controle de problemas de saúde, do impacto físico e psicossocial das doenças e do desenvolvimento de conhecimentos resultantes dos esforços de integração e troca de experiências entre profissionais e pesquisadores sobre esta temática (3).

Estudos sobre a QV de aposentados são escassos e fundamentais, visto que possibilitam identificar os numerosos fatores que permeiam esse processo, favorecendo aos gestores, bem como aos próprios trabalhadores realizarem planejamento visando promover a aposentadoria com mais QV (6).

Além disso, no contexto da aposentadoria, deve-se considerar a aposentadoria por invalidez uma vez que o trabalhador se torna incapacitado definitivamente para o trabalho devido a acidente ou doença (7). A incapacidade permanente motivadora da aposentadoria por invalidez pode estar relacionada à QVT e repercutir na QV no indivíduo.

Assim, este estudo teve como objetivo avaliar a qualidade de vida e os fatores associados em servidores aposentados por invalidez de uma universidade pública.

\section{MATERIAL E MÉTODO}

Estudo transversal e analítico realizado com os servidores aposentados por invalidez entre os anos de 2000 a 2014 de uma universidade pública do Estado do Paraná, Brasil. As aposentadorias do ano de 2015 não foram incluídas devido a indisponibilidade dos dados na universidade. Esta instituição de ensino oferta 54 cursos de graduação e 236 de pós-graduação (residências, especializações, mestrados e doutorados) para 17.858 estudantes. Possui 1.650 docentes e 3.433 profissionais nas demais funções, cargos esses definidos como técnico-administrativos.

Os critérios de elegibilidade estabelecidos foram: ser servidor público estatutário aposentado por invalidez há pelo menos um ano; residir na cidade ou região metropolitana da cidade onde se situa a universidade, em razão do deslocamento para aplicação dos questionários presencialmente, e apresentar capacidade cognitiva para responder aos questionários, isto é, estar orientado auto e alopsiquicamente.

A Pró-Reitoria de Recursos Humanos da universidade em estudo forneceu uma lista com 88 possíveis participantes. Esses aposentados foram convidados para participar da pesquisa por telefonemas, mídias eletrônicas ou visitas em seus domicílios. Após esclarecidos sobre o objeto de investigação e concordarem em participar do estudo foram agendados encontros para a aplicação dos questionários em suas residências. Nesta fase identificou-se que entre os possíveis participantes havia 30 óbitos, 13 perdas (incapacidade para responder aos questionários e não residir na cidade ou região metropolitana) e 5 recusas. Assim, esta investigação foi realizada com 40 aposentados.

A coleta de dados foi realizada pela pesquisadora principal e ocorreu no período de janeiro a junho de 2016. Foram utilizados dois instrumentos, o primeiro foi elaborado 
pelos autores e continha questões sociodemográficas, clínicas, ocupacionais e de hábitos de vida. Entre as variáveis coletadas estavam: sexo, idade, estado civil, nível de instrução, renda familiar, ano que o trabalhador se aposentou da universidade, função exercida, tempo de trabalho na instituição, motivo da aposentadoria por invalidez, presença de comorbidades, a prática de atividade física, tabagismo, uso de bebida alcoólica e lazer. Previamente a coleta, este questionário foi submetido a um pré-teste com 15 aposentados por invalidez da iniciativa privada, com a finalidade de verificar a sua adequação. Após o teste piloto foram acrescentadas alternativas ao turno de trabalho, incluída questão sobre carga horária e ajustes na diagramação para facilitar o preenchimento e a tabulação.

O segundo instrumento foi o questionário genérico de avaliação da QV relacionada à saúde denominado Short Form Health Survey versão 2 (SF-36v2) que teve sua primeira versão desenvolvida na década de 80 nos Estados Unidos e foi traduzido e validado no Brasil na década de 90 (8) e sua segunda versão desenvolvida após a revisão da primeira, ambas as versões condizentes com a realidade brasileira (9).

O SF-36v2 é composto por 36 itens agrupados em 8 domínios: capacidade funcional, aspectos físicos, dor, estado geral de saúde, vitalidade, aspectos sociais, aspectos emocionais, saúde mental e uma questão comparativa sobre a percepção atual da saúde e há um ano. Esses domínios são reagrupados em dois componentes: o físico e o mental. Para a utilização deste questionário foi obtida a autorização do grupo Optum Insight Company QualityMetric sob o no QM032612.

Inicialmente, realizou-se dupla digitação e conferência dos dados. O cálculo dos domínios da escala SF-36v2 foi realizado por meio do programa Quality Metric Health Outcomes $^{T M}$ Scoring Software 4.5, para a ob- tenção da pontuação de 0 a 100, sendo que os maiores escores correspondem a melhor QV relacionada à saúde (10).

O programa Statistical Package for the Social Sciences (SPSS), versão 20.0, foi utilizado para as análises estatísticas. $\mathrm{Na}$ análise descritiva, calculou-se medidas de tendência central e de dispersão para as variáveis numéricas, e frequências absolutas e relativas para as variáveis categóricas. A consistência interna do SF-36v2 foi verificada por meio do coeficiente alfa de Cronbach. $\mathrm{O}$ teste de Kolmogorov-Smirnov indicou que alguns escores do SF-36v2 não apresentavam distribuição normal. Desse modo, para testar a associação entre as dimensões de QV com as variáveis independentes utilizouse o coeficiente de correlação de Spearman e o teste de Mann-Whitney. Na sequência realizou-se regressão linear múltipla, pelo método backward e utilizando a técnica de reamostragem (bootstrap), queérecomendada para amostras pequenas, com o propósito de obter estimativas não enviesadas no modelo multivariado (11). Foram incluídas todas as variáveis que apresentaram $\mathrm{p} \leq 0,20$ na análise bivariada e permaneceram no modelo final o conjunto de variáveis independentes que melhor explicavam o desfecho e mantiveram associação significativa após o ajuste $(p \leq 0,05)$. O modelo múltiplo seguiu as premissas de linearidade, homocedasticidade e ausência de autocorrelação dos resíduos (12).

Este estudo é originário do projeto denominado "Qualidade de vida de servidores aposentados de uma universidade pública”, e seguiu todos os aspectos éticos brasileiros e internacionais de pesquisas envolvendo seres humanos, incluindo aprovação em Comitê de Ética em Pesquisa, parecer no 916.930, bem como todos os participantes assinaram o Termo de Consentimento Livre e Esclarecido. 


\section{RESULTADOS}

Entre os 40 aposentados por invalidez que participaram do estudo a idade média foi de $56,8$ anos ( $\mathrm{DP}=9,7)$, variando entre 39 e 85 anos. A maioria pertencia ao sexo feminino $(72,5 \%$; $n=29)$, possuía união conjugal estável $(67,5 \% ; n=27)$ e ao menos um dependente $(65 \% ; n=26)$. A renda familiar média foi de $\mathrm{R} \$ 4.987,38$ (US\$1.552,44), com mínima de R $\$ 1.200,00$ (US\$373,52) e máxima de $\mathrm{R} \$ 35.000,00$ (US\$10.894,60).

Quanto aos hábitos de vida referiram possuir atividade de lazer $(57,5 \% ; n=23)$ e a minoria afirmou realizar atividade física $(35,0 \% ; n=14)$, tabagismo $(30,0 \% ; n=12)$ e consumo de bebidas alcoólicas $(17,5 \% ; n=7)$.

No que diz respeito às características ocupacionais exercidas anteriormente a aposentadoria, 7,5\% $(n=3)$ eram docentes e $92,5 \%$ $(n=37)$ exerciam atividades técnicas, com dedicação de 40 horas semanais $(75 \% ; n=30)$ ou mais $(25 \% ; n=10)$ e trabalharam em média 16,5 anos na universidade em estudo.

Sobre o tempo de aposentadoria, a média foi 8,4 anos e variou entre 2 e 16 anos. As doenças mentais e comportamentais (DMC) constituíram-se como as maiores responsáveis pela invalidez entre esta população (35\%; $n=14)$. Entre as demais causas de aposentadoria, identificaram-se as doenças osteomusculares $(22,5 \% ; n=9)$, cardiovasculares $(15,0 \% ; n=6)$, neoplasias $(7,5 \% ; n=3)$ e outras causas $(20,0 \% ; n=8)$.

Referente às comorbidades dos participantes, $42,5 \%(n=17)$ relataram depressão, seguido de 37,5\% $(n=15)$ hipertensão arterial sistêmica, $37,5 \%(n=15)$ doenças osteomusculares, $17,5 \%(n=7)$ acidente vascular encefálico, $17,5 \% \quad(n=7)$ neoplasia, $12,5 \%$ $(n=5)$ diabetes mellitus e 2,5\% $(n=1)$ doença neurológica.

Na Tabela 1, a QV está demonstrada por meio dos resultados dos componentes e domínios da escala SF-36v2. Pode-se verificar que nos domínios aspectos sociais e emocionais foram obtidos as maiores pontuações médias e as menores foram encontradas nos domínios vitalidade e dor. Observou-se que nos dois componentes a média esteve abaixo de 50, sendo a menor pontuação a do mental. A análise dos valores de alfa de Cronbach indicou que todos os domínios apresentaram boa consistência interna.

Tabela 1. Medidas descritivas e Alfa de Cronbach do SF36v2 dos aposentados por invalidez de uma universidade pública. Londrina, Paraná, Brasil, 2016.

\begin{tabular}{llccccc}
\hline Componentes e Domínios & Média & $\begin{array}{c}\text { Desvio- } \\
\text { padrão }\end{array}$ & $\begin{array}{c}\text { Mínimo } \\
\text {-Máximo }\end{array}$ & Mediana & $\begin{array}{c}\text { Alfa de } \\
\text { Cronbach }\end{array}$ \\
\hline \multirow{6}{*}{ Capacidade funcional } & 53,5 & 29,6 & $0,0-100,0$ & 52,5 & 0,923 \\
& Aspectos físicos & 53,3 & 36,3 & $0,0-100,0$ & 56,2 & 0,951 \\
& Dor & 48,1 & 31 & $0,0-100,0$ & 46 & 0,86 \\
& Estado geral de saúde & 52,8 & 24,5 & $0,0-92,0$ & 58,5 & 0,766 \\
& Vitalidade & 46,1 & 21,1 & $0,0-87,5$ & 46,9 & 0,652 \\
& Aspectos sociais & 60 & 35,5 & $0,0-100,0$ & 62,5 & 0,836 \\
& Aspectos emocionais & 60 & 31,4 & $0,0-100,0$ & 62,5 & 0,852 \\
& Saúde mental & 52,5 & 25,7 & $0,0-90,0$ & 55 & 0,863 \\
\hline \multirow{2}{*}{ Componentes } & Físico & 42,3 & 12,1 & $17,6-70,2$ & 44 & - \\
& Mental & 41,1 & 16,4 & $9,3-69,1$ & 40 & - \\
\hline
\end{tabular}


Os fatores associados de cada componente e domínio do SF36v2 estão apresentados na Tabela 2, sendo que todos os modelos multivariados apresentaram significância estatística $(\mathrm{p}<0.001) .30,4 \%$ da variação da capacidade funcional e $26 \%$ do componente físico foram explicados pelas variáveis neoplasia e carga horária, assim, os menores escores ocorreram entre os aposentados com neoplasia e que desempenhavam carga horária de trabalho maior que $40 \mathrm{~h}$ quando estavam na ativa. O lazer explicou $10,6 \%$ da variação do domínio aspectos físicos. Os aposentados com diabetes mellitus apresentavam menores pontuações no domínio dor, que explicou $24,8 \%$ de sua ocorrência. $28,1 \%$ da variação dos escores no estado geral de saúde estive- ram associados aos aposentados com neoplasia, doença neurológica e que não possuíam relacionamento conjugal estável. A vitalidade foi menor entre aqueles que indicaram acidente vascular encefálico e doenças osteomusculares, explicando $22,9 \%$ da variação deste domínio. As piores percepções de aspectos sociais, saúde mental e no componente mental foram obtidas pelos aposentados com depressão, que explicou a variação de $40,9,34,2$ e $41,1 \%$, respectivamente. Por fim, o domínio aspectos emocionais foi explicado em 34,3\% pelo motivo da aposentadoria, isto é, aposentar-se por transtornos mentais e do comportamento diminuiu a percepção da QV relacionada aos aspectos emocionais.

Tabela 2. Fatores associados aos componentes e domínios do SF36v2 dos aposentados por invalidez de uma universidade pública. Londrina, Paraná, Brasil, 2016.

\begin{tabular}{|c|c|c|c|c|}
\hline \multicolumn{3}{|c|}{ Componentes e Domínios } & \multirow{2}{*}{$\begin{array}{c}\text { Beta } \\
-32087\end{array}$} & \multirow{2}{*}{$\frac{\text { valor } \mathrm{p}}{0,001}$} \\
\hline \multirow{12}{*}{ Dominios } & \multirow{2}{*}{ Capacidade Funcional $\left(\mathrm{R}^{2}=0,304\right)$} & Neoplasia & & \\
\hline & & Carga horária & $-0,535$ & 0,002 \\
\hline & Aspectos Físicos $\left(\mathrm{R}^{2}=0,106\right)$ & Lazer & 23609 & 0,04 \\
\hline & Dor $\left(\mathrm{R}^{2}=0,248\right)$ & Diabetes mellitus & -37159 & 0,001 \\
\hline & \multirow{3}{*}{ Estado Geral de Saúde $\left(\mathrm{R}^{2}=0,281\right)$} & Neoplasia & -20758 & 0,021 \\
\hline & & Doenças neurológicas & -53261 & 0,002 \\
\hline & & Estado civil & 16760 & 0,028 \\
\hline & \multirow{2}{*}{ Vitalidade $\left(\mathrm{R}^{2}=0,229\right)$} & Acidente vascular encefálico & -17552 & 0,005 \\
\hline & & Doenças osteomusculares & -18626 & 0,007 \\
\hline & Aspectos Sociais $\left(\mathrm{R}^{2}=0,409\right)$ & Depressão & -35888 & 0,052 \\
\hline & Aspectos Emocionais $\left(\mathrm{R}^{2}=0,343\right)$ & Motivo da aposentadoria & -25862 & 0,026 \\
\hline & Saúde Mental $\left(\mathrm{R}^{2}=0,342\right)$ & Depressão & -20508 & 0,013 \\
\hline \multirow{3}{*}{ Componentes } & \multirow{2}{*}{ Físico $\left(\mathrm{R}^{2}=0,260\right)$} & Neoplasia & -10167 & 0,018 \\
\hline & & Carga horária & $-0,243$ & 0,003 \\
\hline & Mental $\left(\mathrm{R}^{2}=0,411\right)$ & Depressão & -13542 & 0,012 \\
\hline
\end{tabular}

\section{DISCUSSÃO E CONCLUSÃO}

As características sociodemográficas dos participantes deste estudo mostraram-se semelhante às de outras investigações, quanto a maior frequência do sexo feminino, acometimento de trabalhadores não idosos e menor escolaridade (13-16). Entretanto, a predominância de aposentados com relacionamentos conjugais e a renda mensal média foi diferente daquelas obtidas por outros estudos (13-14). 
A maior frequência de profissionais aposentados na carreira técnica era esperada devido ao maior contingente destes trabalhadores, pois estes ocupam os demais cargos na universidade excluindo a docência, evidenciado também em investigação realizada com servidores públicos aposentados de uma universidade federal do Brasil (13). Sobre a carga horária exercida, apesar de todos os servidores aposentados apresentarem o mesmo vínculo estatutário, houve diferença nas horas trabalhadas, isto pode estar relacionado às funções exercidas com cargas horárias diferentes, bem como a realização de horas extras devido à falta de profissionais para manter os serviços da instituição. Esta diferença entre a carga horária de servidores públicos também foi encontrada em um estudo realizado com servidores técnico-administrativos de outra universidade pública brasileira (17).

As DMC, como motivos de aposentadoria por invalidez, constituem-se na primeira causa em investigações tanto a nível nacional como internacional $(13,14,16,18,19)$, dados esses que são análogos aos encontrados no presente estudo.

As doenças crônicas (DC) são um problema de saúde mundial, ocasionando complicações, incapacidades e mortes, além dos custos dispendidos. A carga de DC está relacionada a fatores preveníveis como dieta inadequada, tabagismo, inatividade física e consumo excessivo de álcool (20). Essa realidade coincide com os resultados deste estudo uma vez que foi encontrada alta frequência das DC, bem como a não adesão à atividade física e dieta alimentar. Além disso, a maioria referiu não fumar e não ingerir bebidas alcoólicas. Contudo estes hábitos podem ter sido ajustados após surgimento de alguma DC e diante da necessidade de uso de medicação contínua.

É fato que as morbidades estão diretamente relacionadas à deterioração da $\mathrm{QV}$, conforme o aumento das comorbidades há uma diminuição na QV avaliada pelo ques- tionário SF-36v2 $(21,22)$. Isso está confirmado neste estudo pelas associações inversamente proporcionais da QV com a presença das comorbidades: neoplasia, depressão, diabetes mellitus e doenças neurológicas, evidenciando que medidas de promoção e prevenção necessitam ser tomadas entre a população geral.

Quanto aos dados normativos da escala SF-36v2 no Brasil, quase todos os escores obtidos são menores que os encontrados entre a população de outros países como: Canadá (23) e Estados Unidos (10). Ao comparar os dados normativos da escala SF-36v2 com os escores dos aposentados por invalidez da presente investigação, identifica-se que a maioria dos domínios apresentam escores bem inferiores à média nacional (24). Diante disso, pode-se afirmar que os aposentados por invalidez deste estudo apresentam uma QV insatisfatória, uma vez que os componentes físico e mental apresentam escores abaixo de 50. Além disto, os domínios com maiores pontuações obtiveram médias que estiveram próximas a este valor.

Ao analisar a carga horária trabalhada anteriormente pelos aposentados por invalidez, foi identificado que quanto maior essa carga horária, maior o comprometimento do componente físico e do domínio capacidade funcional. A jornada de trabalho excessiva associada à inatividade física, prevalência de sobrepeso e obesidade, alimentação inadequada, além de menor tempo de sono, contribui para a deterioração da saúde do trabalhador e reflete na $\mathrm{QV}$, ainda mais na aposentadoria por invalidez, já que estes hábitos podem ter resultado na doença incapacitante (25).

A depressão interferiu negativamente no componente mental, nos domínios aspectos social e saúde mental, em que são avaliadas a interferência dos problemas de saúde e emocionais nos relacionamentos e como sentiuse nas quatro semanas anteriores, quando comparada as outras doenças a depressão apresenta menores escores no componente mental, inclusive sendo composto pelos 
domínios encontrados na associação (26). Ainda, o motivo da aposentadoria que se associou inversamente aos escores dos aspectos emocionais, ou seja, as DMC prejudicaram o desempenho dos aposentados por invalidez nas relações sociais e na realização de atividades diárias decorrentes aos problemas emocionais avaliados neste domínio. Isso coincide com uma investigação brasileira em que se constatou que a presença de doenças mentais foi associada às menores pontuações em todos os domínios avaliados pelo SF-36 (27).

Os únicos fatores que contribuíram diretamente para uma melhor QV foram os relacionamentos conjugais estáveis e o lazer, corroborando com outro estudo brasileiro em que as melhores pontuações nos domínios do SF-36v2 estiveram correlacionadas aos aspectos físicos, estado geral de saúde, vitalidade, aspectos sociais e saúde mental (28). $\mathrm{Na}$ investigação internacional realizada com pessoas turcas, os idosos casados também apresentaram maiores pontuações relacionadas à QV (29).

As oportunidades de lazer entre os aposentados por invalidez contribuíram para uma melhor QV somente no domínio dos aspectos físicos, e este está relacionado à interferência dos problemas de saúde nas atividades cotidianas e disposição para realização destas. O lazer contribui para melhora de muitos aspectos na vida das pessoas, como na melhor percepção da $\mathrm{QV}$, inclusive a atividade física no lazer pode contribuir para diminuição da intensidade da dor e na melhora da função física e mental $(25,30,31)$.

Diante do exposto, é essencial implementar medidas para controle das doenças, estímulo à hábitos de vida saudáveis e promoção de saúde, para melhoria da QV desta população, bem como a prevenção de outros agravos à saúde das pessoas que já se encontra comprometida. Também, é importante que se realize planejamentos em conjunto com os gestores e trabalhadores quando ainda estiveram exercendo suas atividades laborais com o objetivo de prevenir doenças, e, por sua vez, aposentadorias precoces motivadas pela invalidez.

Sugere-se que sejam adotadas medidas de promoção de saúde dentro dos locais de trabalho em parceria com os diferentes centros de estudo da universidade por meio de projetos de extensão, inclusive da área da enfermagem, voltado para promoção de saúde dos servidores e aposentados da instituição com a finalidade de promover a QV e QVT.

A presente pesquisa apresentou limitações quanto ao delineamento transversal, que impede de direcionar as relações causais, assim como o número de participantes do estudo. Vale destacar que o maior número de perdas decorreu dos óbitos, revelando um problema importante para análises futuras. Ainda assim, os resultados encontrados podem subsidiar na elaboração de planejamentos das políticas de atenção à saúde dos servidores de universidades públicas, e também pode ser a base para o desenvolvimento de outras investigações sobre a temática.

Os resultados permitem afirmar que a população de aposentados por invalidez deste estudo apresentou uma QV insatisfatória, visto que, as pontuações do SF-36v2 estiveram muito próximas de 50 e as medidas sumárias dos componentes mental e físico estiveram abaixo deste valor.

Os fatores associados diretamente a uma melhor avaliação da QV estiveram presentes no que concerne ao relacionamento conjugal estável e atividades de lazer. As DC foram as principais responsáveis pela associação com uma pior QV entre os aposentados por invalidez.

\section{REFERÊNCIAS}

1. Pilger C, Dias JF, Kanawava C, Baratieri T, Carreira L. Compreensão sobre o envelhecimento e ações desenvolvidas pelo enfermeiro na atenção primária à saúde. Cienc enferm. 2013; XIX(1): 61-73. 
2. Yeung DY, Zhou X. Planning for Retirement: Longitudinal Effect on Retirement Resources and Post-retirement Well-being. Front Psychol. 2017; 8:1300.

3. Minayo MCS, Hartz ZMA, BUSS PM. Quality of life and health: a necessary debate. Cien Saude Colet. 2000; 5(1): 7-18.

4. World Health Organization Quality of Life Group. The World Health Organization quality of life assessment: development and general psychometric properties. Soc Sci Med. 1998; 46(12): 1569-85.

5. Ferreira Junior RR, Porto AP. La calidad de vida en el trabajo y el ausentismo como indicadores de resultado de gestión hospitalaria. Ciencias Administrativas. 2017; 1(11): 4-14.

6. Liberatti VM, Martins JT. Social representations of retirement for healthcare nurses of a public university. J Nurs UFPE. 2012; 6(10): 2603-5.

7. Silveira JAC. The concept of disability in the sphere of disability retirement benefit. Rev Direito Liberdade. 2015;17(1): 91-130.

8. Ciconelli RM, Ferraz MB, Santos W, Meinão I, Quaresma MR. Brazilian-Portuguese version of the SF-36. A reliable and valid quality of life outcome measure. Rev Bras Reumatol. 1999; 39: 14350.

9. Campolina AG, Ciconelli RM. O SF-36 e o desenvolvimento de novas medidas de avaliação de qualidade de vida. Acta Reumatol Port. 2008; 33(2): 127-33.

10. Maruish ME. Users's manual for the SF$36 v 2$ Health Survey. $3^{\text {rd }}$ edition. Lincoln: Quality Metric Incorporated; 2011.

11. Silva Filho AS. Inferência em amostras pequenas: método bootstrap. Rev Ciênc Exatas Tecnologia. 2010; 5(5): 115-126.

12. Leech NL, Barrett KC, Morgan GA. SPSS for Intermediate Statistics: use and interpretation. $5^{\text {th }}$ edition. New York: Routledge; $2012.382 \mathrm{p}$.

13. Santos ACQ, Limongi JE, Jorge MLMP, Jorge MT, Pereira BB, Jorge PT. Retire- ment for disability and chronic diseases among workers at the Municipality of Uberlândia, Minas Gerais, 1990-2009. Cad Saude Colet. 2015; 23(1): 57-62.

14. Brites RMR, Abreu AMM, Pinto JESS. Prevalence of alcoholism in the profile of disability retirement among employees of a federal university. Rev Bras Enferm. 2014; 67(3): 373-80.

15. Halford C, Wallman T, Welin L, Rosengren A, Bardel A, Johansson S, et al. Effects of self-rated health on sick leave, disability pension, hospital admissions and mortality. A population-based longitudinal study of nearly 15,000 observations among Swedish women and men. BMC Public Health. 2012; 12: 1103.

16. Narusyte J, Ropponen A, Silventoinen K, Alexanderson K, Kaprio J, Samuelsson A, et al. Genetic liability to disability pension in women and men: a prospective population-based twin study. PLos ONE [Internet]. 2011 [citado 12 fev 2017]; 6(8): e23143. Disponível em: http://journals. plos.org/plosone/article?id=10.1371/ journal.pone.0023143\#s1

17. Godinho MR, Greco RM, Teixeira MTB, Teixeira LR, Guerra MR, Chaoubah A. Work ability and associated factors of Brazilian technical-administrative workers in education. BMC Res Notes. 2016; 9: 1 .

18. Martins JT, Galdino MJQ, Linares PG, Ribeiro RP, Ueno LGS, Bobroff MCC. Disability retirement of workers in the health field at a university hospital. J Res Fundam Care Online. 2017; 9(1): 122-7.

19. Kaila-Kangas L, Haukka E, Miranda H, Kivekas T, Ahola K, Luukkonen R, et al. Common mental and musculoskeletal disorders as predictors of disability retirement among Finns. J Affect Disord. 2014; 35: 38-44.

20. Bauer UE, Briss PA, Goodman RA, Bowman BA. Prevention of chronic disease in the 21st century: Elimination of the leading preventable causes of premature 
death and disability in the USA. The Lancet. 2014; 84(9937): 45-52.

21. Maciel NM, Conti MHS, Simeão SFAP, Corrente JE, Ruiz T, Vitta A. Reported morbidities and quality of life: population-based study. Fisioter Pesq. 2016; 23(1): 91-7.

22. Adriaanse MC, Drewes HW, Van der Heide I, Struijs JN, Baan CA. The impact of comorbid chronic conditions on quality of life in type 2 diabetes patients. Qual Life Res. 2016; 25:175.

23. Hopman WM, Towheed T, Anastassiades T, Tenenhouse A, Poliquin S, Berger C et al. Canadian normative data for the SF-36 health survey. Canadian Multicentre Osteoporosis Study Research Group. CMAJ. 2000; 163(3): 265-71.

24. Laguardia J, Campos MR, Travassos C, Najar AL, Anjos LA, Vasconcellos MM. Brazilian normative data for the Short Form 36 questionnaire, version 2. Rev Bras Epidemiol. 2013; 16(4): 889-97.

25. Fernandes JC, Portela LF, Rotenberg L, Griep RH. Working hours and health behaviour among nurses at public hospitals. Rev Lat Am Enfermagem. 2013; 21(5): 1104-11.

26. Saragoussi D, Christensen MC, Hammer-Helmich L, Rive B, Touya M, Haro JM. Long-term follow-up on health-related quality of life in major depressive disorder: a 2-year European cohort study. Neuropsychiatric Dis Treat. 2018; 14: 1339-50.

27. Jansen K, Mondin TC, Ores LC, Souza LDM, Konradt CE, Pinheiro RT et al. Mental common disorders and quality of life in young adulthoods: a population-based sample in Pelotas, Rio Grande do Sul State, Brazil. Cad Saude Publica. 2011; 27(3): 440-8.

28. Andrade JMO, Rios LR, Teixeira LS, Vieira FS, Mendes DC, Vieira MA et al. Influence of socioeconomic factors on the quality of life of elderly hypertensive individuals. Cienc Saude Colet. 2014; 19(8): 3497-504.

29. Erkal S, Sahin H, Surgit EB. Examination of the relationship between the quality of life and demographic and accident-related characteristics of elderly people living in a nursing home. Turk J Geriat. 2011; 14: 45-53.

30. Geneen LJ, Moore R, Clarke C, Martin D, Colvin LA, Smith BH. Physical activity and exercise for chronic pain in adults: an overview of Cochrane Reviews. Cochrane Database of Syst Rev. 2017; 4(011279).

31. Vitorino LM, Paskulin LMG, Vianna LAC. Quality of life of seniors living in the community and in long term care facilities: a comparative study. Rev Lat Am Enfermagem. 2013; 21(spe): 3-11. 